\title{
Anomalous Capacitance Maximum of the Glassy Carbon-Ionic Liquid Interface through Dilution with Organic Solvents
}

\author{
David J. Bozym, ${ }^{\dagger}$ Betül Uralcan, ${ }^{\dagger}$ David T. Limmer, ${ }^{\dagger}$ Michael A. Pope, ${ }^{\dagger}$ Nicholas J. Szamreta, ${ }^{\dagger}$ \\ Pablo G. Debenedetti, ${ }^{\dagger}$ and Ilhan A. Aksay* ${ }^{\dagger}$ \\ ${ }^{\dagger}$ Department of Chemical and Biological Engineering, Princeton University, Princeton, New Jersey 08544, United States \\ ${ }^{\ddagger}$ Princeton Center for Theoretical Science, Princeton University, Princeton, New Jersey 08540, United States
}

\section{Supporting Information}

ABSTRACT: We use electrochemical impedance spectroscopy to measure the effect of diluting a hydrophobic room temperature ionic liquid with miscible organic solvents on the differential capacitance of the glassy carbon-electrolyte interface. We show that the minimum differential capacitance increases with dilution and reaches a maximum value at ionic liquid contents near 5$10 \mathrm{~mol} \%$ (i.e., $\sim 1 \mathrm{M}$ ). We provide evidence that mixtures with 1,2-dichloroethane, a lowdielectric constant solvent, yield the largest gains in capacitance near the open circuit potential when compared against two traditional solvents, acetonitrile and propylene carbonate. To provide a fundamental basis for these observations, we use a coarse-grained model to relate structural variations at the double layer to the occurrence of the maximum. Our results reveal the potential for the enhancement of double-layer capacitance through dilution.

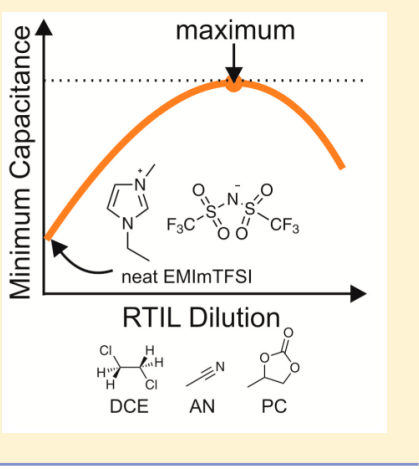

$\mathrm{R}$ oom temperature ionic liquids (RTILs) are regarded as the next-generation electrolytes for electrochemical double-layer capacitors (EDLCs), as their low volatility and wide electrochemical windows $(>4 \mathrm{~V})$ can lead to safer devices with greater energy density. ${ }^{1}$ However, the low conductivity of RTILs limits the rate performance of EDLCs and yields devices with suboptimal power density. ${ }^{2}$ To overcome this limitation, in top-performing EDLCs, $50 \mathrm{wt} \%$ acetonitrile (AN) is added to the RTIL electrolyte $(\sim 1 \mathrm{M})$, as the bulk conductivity of the mixture is greater than that of the neat RTIL due to a lower viscosity. ${ }^{3-5}$ Such RTIL/solvent mixtures are electrochemically stable up to $4 \mathrm{~V}$ and have also shown promise in devices operating at high temperatures. ${ }^{6,7}$ Additionally, RTILs offer processing advantages over traditional solid salt-based electrolytes, namely, they can be evaporatively consolidated with the active electrode material from a volatile phase (e.g., organic solvent) to form dense electrodes. ${ }^{3,8}$ Though improvements in rate performance have been achieved using these mixtures, the effect of diluting RTILs with organic solvents on the doublelayer capacitance of the electrode-electrolyte interface remains unclear. $^{9-12}$ Understanding the effect of RTIL dilution with organic solvents on the double-layer capacitance is critical in the design of RTIL/solvent combinations that maximize capacitance and, correspondingly, improve the energy density of EDLCs.

A large body of work exists regarding the theory and characterization of neat RTILs; yet, only a few reports have studied RTIL/solvent mixtures. ${ }^{13}$ Recently, several computational works have suggested that the addition of nonionic solvent (to $\sim 1 \mathrm{M}$ ) to RTILs has an insignificant effect on the double-layer capacitance. ${ }^{9,10}$ This claim runs counter to the Gouy-Chapman-Stern (GCS) theory of the electrochemical double-layer (EDL) as a decrease in capacitance with dilution is expected, due to a decreasing ion concentration. ${ }^{14}$ Furthermore, two previous experimental studies suggest a maximum in capacitance with dilution. ${ }^{15,16}$ Liu et al. show a maximum massspecific capacitance at $2.0 \mathrm{M}$ 1-ethyl-3-methylimidazolium tetrafluoroborate in $\mathrm{AN}$, yet they are unable to extract information about the double-layer capacitance due to the complicating transport effects of their porous electrodes. ${ }^{15}$ In a second study, Siinor et al. show that the differential capacitance of neat 1-butyl-1-methylpyridinium tetrafluoroborate is less than that of a $0.5 \mathrm{M}$ solution in $\mathrm{AN}$; yet, they do not investigate the technologically relevant concentration regime $(>1 \mathrm{M})$ and overlook the presence of a maximum. ${ }^{16}$ Thus, as a maximum in capacitance with RTIL dilution is not predicted by current EDL theory and as detailed experimental data regarding the doublelayer capacitance for technologically relevant concentration regimes are lacking, our goal, in this study, has been to provide a rigorous analysis of the effect of RTIL dilution with organic solvents on the differential capacitance of the glassy carbon (GC)-electrolyte interface and also provide a fundamental basis for the cause of this effect by using an Ising model.

We use electrochemical impedance spectroscopy (EIS) and a planar GC electrode to probe the EDL and provide insight into the double-layer capacitance using mixtures of RTILs and organic solvents over a wide concentration range (0.1 to 3.85 $\mathrm{M})$. EIS measurements were performed from the open circuit potential (OCP) as suggested by Lockett et al. to reduce hysteresis effects $^{17}$ (see Supporting Information (SI)). We

Received: April 30, 2015

Accepted: June 18, 2015

Published: June 18, 2015 
selected GC as the electrode material, as it not only resembles the disordered carbonaceous materials used in $\mathrm{EDLCs}^{18}$ but also possesses a large space charge capacitance, which allows us to probe the ionic side of the interface with greater sensitivity than if we had used highly oriented pyrolytic graphite, which is limited by its low space charge capacitance. ${ }^{19}$ We also chose the RTIL 1-ethyl-3-methylimidazolium bis(trifluoromethylsulfonyl)imide (EMImTFSI), as it remains popular in the EDLC literature due to its moderate voltage window, relatively low viscosity, and miscibility with a variety of organic solvents. $^{20}$ Using this system, we show that the differential capacitance of the electrode-RTIL interface reaches a maximum with RTIL dilution using three different solvents and provide evidence that this capacitance maximum is not quantitatively correlated with the mixture's bulk conductivity. Furthermore, we show that mixtures with 1,2-dichloroethane (DCE) outperform those with $\mathrm{AN}$, as mixtures with DCE yield the largest gains in capacitance near the OCP, suggesting that AN may not be the best choice of diluent to maximize the double-layer capacitance of the interface. Lastly, we use a coarse-grained model to provide an explanation for this anomalous capacitance maximum in the context of current understanding of the EDL for RTILs.

The minimum differential capacitance $\left(C_{\min }\right)$ of the GCelectrolyte interface for mixtures of EMImTFSI diluted with AN, DCE, and propylene carbonate (PC) is reported in Figure 1a as a function of EMImTFSI content. The $C_{\min }$ for neat EMImTFSI (100 mol\%, $3.85 \mathrm{M})$ is $9.5 \mu \mathrm{F} / \mathrm{cm}^{2}$, a value comparable to what has been previously reported in the literature. ${ }^{21}$ When EMImTFSI is diluted with each solvent, $C_{\text {min }}$ increases and reaches a maximum at an EMImTFSI content
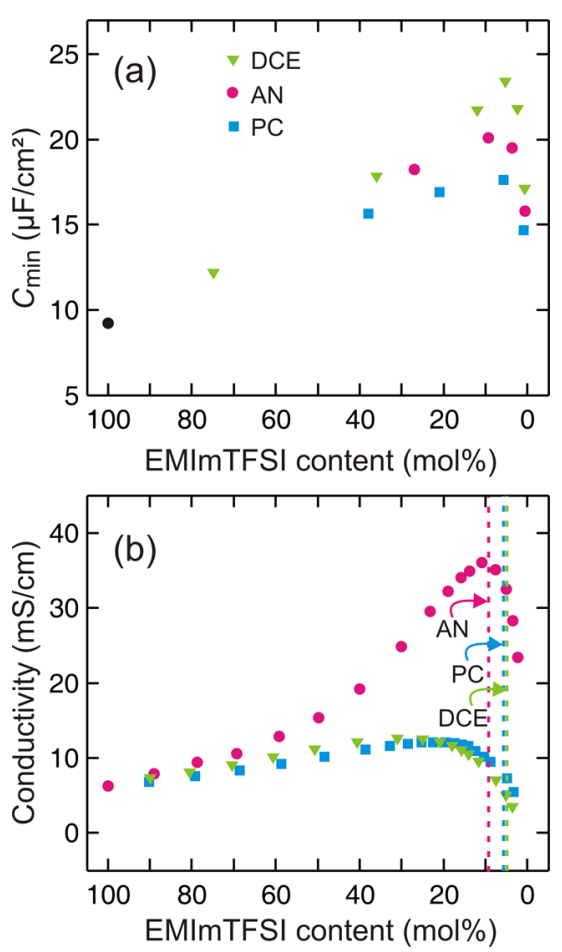

Figure 1. (a) $C_{\min }$ as a function of molar EMImTFSI content for mixtures with AN, DCE, and PC. (b) Bulk conductivity of EMImTFSI/solvent mixtures as a function of EMImTFSI content. The corresponding EMImTFSI content at which the maximum $C_{\min }$ is observed is shown by a dotted line. between 5 and $10 \mathrm{~mol} \%$. This maximum occurs for mixtures of EMImTFSI with each of the three solvents, confirming its existence and demonstrating that the behavior is not exclusive to mixtures with AN. A maximum is unexpected, as the GCS treatment of the EDL predicts that the capacitance should always increase with increasing ion concentration in solvent due to (i) a decrease in the Debye length of the diffuse double-layer and (ii) an increase in the specific adsorption of ions. ${ }^{14}$ In addition to the maximum $C_{\min }$, we note that the absolute values of the maximum $C_{\min }$ for each solvent series are quantitatively different. Mixtures with DCE display the largest maximum $C_{\min }$ of $23.4 \mu \mathrm{F} / \mathrm{cm}^{2}$ at $5.3 \mathrm{~mol} \%$ EMImTFSI, while dilutions of EMImTFSI with AN or PC display maxima of 20.1 and 17.6 $\mu \mathrm{F} / \mathrm{cm}^{2}$, respectively.

In changing the composition of the RTIL/solvent mixture, we note that we are also altering the bulk conductivity of the electrolyte. This has been the primary motivation for using RTIL/solvent mixtures as electrolytes. ${ }^{22}$ A correlation between the conductivity and the double-layer capacitance is unexpected, as the double-layer capacitance is a quantity defined at thermodynamic equilibrium, while bulk conductivity is a kinetic measurement based on dissipative losses in the system. However, the varying solution resistance may have an effect on the EIS measurements. To confirm that the trends in Figure 1a are not an artifact of conductivity, we measured the bulk conductivity of EMImTFSI/solvent mixtures as a function of EMImTFSI content, shown in Figure 1b. For each RTIL/ solvent series, conductivity increases as EMImTFSI is diluted to $\sim 20 \mathrm{~mol} \%$, after which a maximum in conductivity is observed and is expected due to a trade-off between a decreasing viscosity and decreasing ionic concentration with dilution. Mixtures with AN yield the largest values of conductivity, with a maximum of $36.1 \mathrm{mS} / \mathrm{cm}$ observed at $10.8 \mathrm{~mol} \%$ EMImTFSI. This value is nearly three times larger than the conductivity maxima observed for mixtures with DCE and PC, yet mixtures with DCE yield the largest $C_{\text {min }}$. Additionally, the EMImTFSI contents at which the maxima in conductivity and $C_{\min }$ are observed do not coincide for mixtures with DCE and PC. Similarly, the trends in the maximum $C_{\min }$ also do not follow the quantitative trends in the bulk viscosities of the mixtures (Figure S1). Therefore, any apparent, qualitative correlation between conductivity and differential capacitance likely stems from more fundamental, underlying molecular mechanisms such as specific solvent-EMImTFSI interactions. Given these data, we attest that the trends reported in Figure la are not artifacts of mixture conductivity as significant quantitative discrepancies exist between the maximum conductivity and maximum $C_{\min }$ reported for each solvent series.

In addition to the quantitative differences in the maximum $C_{\text {min }}$ the shapes of the differential capacitance-potential ( $C-$ $V$ ) curves of the EMImTFSI/solvent mixtures are remarkably different. Figure 2 shows the $C-V$ curves for neat EMImTFSI as well as for EMImTFSI diluted with each of the three solvents. As seen in Figure 2a, the shape of the $C-V$ curve for neat EMImTFSI is concave up within the $2 \mathrm{~V}$ potential window studied, which is in agreement with the shape predicted by models of nonspherical RTIL ions. ${ }^{23}$ When EMImTFSI is diluted with DCE to $75 \mathrm{~mol} \%$, the capacitance increases globally as shown by the vertical shift in the $C-V$ curve and features a shallow " $U$ "-shape within the given potential window, with a pronounced $C_{\min }$. As EMImTFSI is further diluted to 36 mol\%, the slopes of the anodic and cathodic branches of the $C-V$ curve increase, and the curve assumes a sharper "V"- 

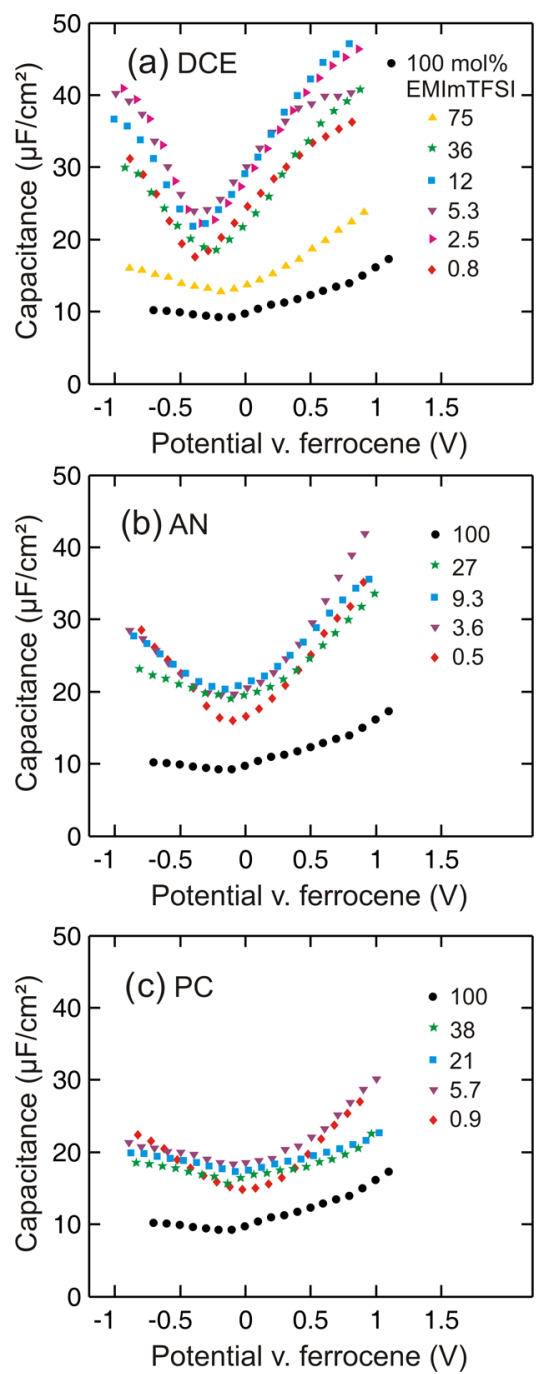

Figure 2. Differential capacitance-potential curves of the GCelectrolyte interface using neat EMImTFSI and EMImTFSI diluted with (a) DCE, (b) AN, and (c) PC.

shape, which is retained at $0.8 \mathrm{~mol} \%$ EMImTFSI. Similar behavior is observed in Figure $2 b$ for mixtures of EMImTFSI/ $\mathrm{AN}$, as the slopes of the anodic and cathodic branches increase as EMImTFSI is diluted to $0.5 \mathrm{~mol} \%$. However, the increase in the slopes is smaller than that observed for the EMImTFSI/ DCE mixtures. For the EMImTFSI/PC mixtures shown in Figure $2 \mathrm{c}$, the increase in the slopes is minimal up to $21 \mathrm{~mol} \%$, and only at $5.7 \mathrm{~mol} \%$ does a shallow "U"-shape appear. Thus, the gains in differential capacitance of EMImTFSI/solvent mixtures near the OCP increase in the order of $\mathrm{PC}<\mathrm{AN}<$ DCE. This result is remarkable, as it suggests that a lowdielectric constant solvent, such as DCE $(\varepsilon \sim 11)$ can yield greater enhancement in double-layer capacitance than AN ( $\varepsilon$ 234) and, as a result, may serve as a more useful diluent for high energy density EDLCs. ${ }^{24}$

While concentration effects in dilute solutions are well understood, i.e., the GCS theory predicts the capacitance increases proportionally to the square root of the bulk ion concentration, ${ }^{14}$ a similar understanding for RTILs is lacking in view of the experimental results presented above. To gain insight into the role of dilution on the capacitance in EMImTFSI, we have simulated a coarse-grained model, defined by the Hamiltonian

$$
H[\{\mathbf{s}\}]=-J \sum_{\langle i, j\rangle} s_{i} s_{j}+\frac{q^{2}}{2} \sum_{i \neq j} s_{i} s_{j} v\left(\mathbf{r}_{i j}\right)
$$

where $q$ is the charge density of a lattice site, $J$ sets the strength of local repulsive interactions, $\{\boldsymbol{s}\}$ denotes the vector of Isinglike variables, $s_{i}=[0, \pm 1]$, the bracket $\langle i j\rangle$ denotes a restriction over distinct nearest neighbor pairs, $\boldsymbol{r}_{i j}$ is the distance between sites $i$ and $j$ on a three-dimensional cubic lattice, and $v(r)$ is a Coulomb interaction evaluated on those lattice sites, which approaches $1 /|r|$ as $r \rightarrow \infty$. This so-called charge-frustrated Ising model ${ }^{25}$ has been studied theoretically in the context of ionic liquids, reproducing interfacial experimentally observed phase diagrams, as well as molecular models. ${ }^{26}$ In this model, solvent molecules are idealized as noninteracting cells, $s_{i}=0$, with unit dielectric constant, and ions are constrained to a fixed density, $\rho$.

Capacitance calculations for $J=1.5$ and $q=2$ are shown in Figure 3 with additional calculations and simulation details
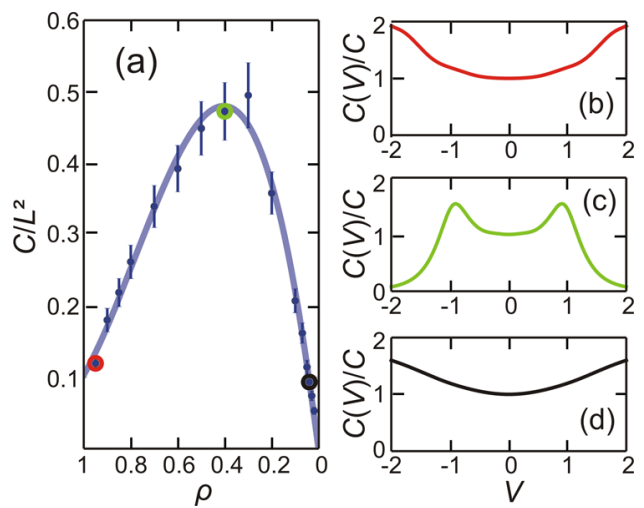

Figure 3. Capacitance as a function of ion concentration and potential for the charge-frustrated Ising model. (a) Capacitance as a function of ion concentration, for $q=2$ and $J=1.5$, normalized by the area of the electrode, $L^{2}$. Error bars denote 1 standard deviation, and the line is a guide to the eye that asymptotes to $\sqrt{ } \rho$ for small $\rho$. (b-d) Capacitance curves for three concentrations, (b) $\rho=0.95$, (c) 0.4 , and (d) 0.05 , as labeled by additional markers in panel a.

available in the SI. Consistent with experiment, for certain parameter regimes, we find a nonmonotonic dependence of the capacitance at zero charge, $C$, as a function of ion concentration. While for low ion concentration we recover the square root dependence predicted by GCS theory, deviations from this scaling are found for $\rho>0.1$. Figure $3 \mathrm{~b}-$ $\mathrm{d}$ shows that the capacitance as a function of applied voltage, $C(V)$, is similarly consistent with the experimental measurements, with a curvature around $V=0$ that increases with increasing ion concentration. For all $\rho>0.1$, we find a double peak forms in the $C V$ curves, as has been observed previously, ${ }^{23}$ with an increasing voltage range between the two peaks observed with increasing concentration.

Within this model, the capacitance maximum results from a competition between charge fluctuations at the electrode due to the uncorrelated motion of single free ions that dominates in the dilute regime, and those similarly free motions of dilute solvent molecules that can dominate in the highly concentrated limit. While the solvent molecules themselves do not carry a charge, due to the incompressibility of the lattice, solvent motion in a concentrated electrolyte occurs with the correlated swapping of a charge in the direction opposing its motion. 
When solvent molecules are dilute, they exhibit exponential correlations away from the electrode and concurrently increase the capacitance proportionally to the square root of their concentration, just as dilute ions do within the context of GCS theory. When these solvent-induced charge fluctuations are large compared to typical charge fluctuations within the ionic liquid, such as for large $q$ where the liquid interface is highly charge-ordered, the capacitance will increase with dilution. Alternatively, when ion fluctuations are facile, the capacitance can remain constant or even increase with increasing concentration (see SI). The dependence of the capacitance maximum with dielectric constant, observed in the experiments, can be interpreted within this mechanism as reflecting the size of the effective charge correlated with solvent motion, with higher dielectric constant solvents reducing the magnitude of this charge through screening and subsequently reducing the capacitance. Recently, experimental studies have also suggested that the interfacial structures of RTILs differ considerably from the bulk and feature charge-ordered or "solid-like" behavior, ${ }^{27,28}$ which is consistent with the findings of this coarsegrained model. Understanding how molecular features of the solvent and ions such as size, shape, and polarizability result in changes to these effective parameters requires molecular level models and is worth further exploration.

Our results confirm that the differential capacitance of the electrode-electrolyte interface can, in fact, increase through diluting EMImTFSI with a miscible organic solvent to EMImTFSI contents between 5 and $10 \mathrm{~mol} \%$, depending on the solvent used. Additionally, we demonstrate that of the three solvents studied, DCE, a low-dielectric constant solvent, yields the greatest gains in capacitance at all potentials studied, suggesting that AN may not be the optimal solvent for high energy density EDLCs. Such knowledge may ultimately prove useful in engineering new RTIL-solvent mixtures for EDLCs, which maximize both power and energy density.

\section{EXPERIMENTAL METHODS}

AN (99.8\%, Sigma-Aldrich), DCE (99.8\%, Sigma-Aldrich), and PC (99.7\%, Sigma-Aldrich) were stored in a glovebox (Innovative Technologies, $\mathrm{H}_{2} \mathrm{O}<1.0 \mathrm{ppm}, \mathrm{O}_{2}<0.2 \mathrm{ppm}$ ) filled with ultrapure argon (99.9999\%, AirGas) immediately upon delivery in sealed containers. EMImTFSI (99.5\%, Iolitec) was purchased at its highest available purity. The as-received RTIL was further purified by washing 5 times with warm, deionized water and drying at $110^{\circ} \mathrm{C}$ under vacuum for 2 days on a standard Schlenk line. The purified RTIL was immediately transferred to the glovebox for storage until use. Figure S2 shows cyclic voltammograms of EMImTFSI before and after purification. Electrolyte mixtures were prepared gravimetrically and vigorously mixed inside the glovebox using a Teflon stir-bar for $15 \mathrm{~min}$ prior to use. Glass pipettes were used, and extreme care was taken not to contaminate the RTIL or solvents during preparation. Each mixture was freshly prepared before use.

A $3 \mathrm{~mm}$ diameter $\mathrm{GC}$ rod electrode ( $\mathrm{CH}$ Instruments) was polished with $0.05 \mu \mathrm{m}$ aluminum monohydroxide powder, rinsed with deionized water and bath sonicated in ethanol for $20 \mathrm{~min}$ after each polish. The rod electrode was immediately transferred to the glovebox, where it remained until use. A platinum mesh was used as a counter electrode, while a pseudoreference electrode was employed consisting of a platinum wire encased in a fritted glass sheath and filled with the identical electrolyte composition as probed in each measurement. The reference electrode architecture allowed for isolation of the platinum wire from the bulk electrolyte and kept the wetted area of the wire constant. Immediately after each measurement was performed, the reference electrode was calibrated against a $5 \mathrm{mM}$ ferrocene standard for the same $\mathrm{RTIL} /$ solvent composition of the analyzed electrolyte; ferrocene is not present during the impedance measurements. The ferrocene electrolyte was also used to confirm the geometric surface area of the electrode using the Cottrell equation, ${ }^{29}$ which was $0.071 \pm 0.002 \mathrm{~cm}^{2}$.

Cyclic voltammetry and EIS were performed using a computer-controlled digital potentiostat (VSP, Bio-Logic, USA). All experiments were conducted in a three-electrode electrochemical cell inside the glovebox. Cyclic voltammetry was performed at $10 \mathrm{mV} / \mathrm{s}$ three times across the selected voltage window to clean the electrode surface prior to measurements. Impedance measurements were taken from 10 $\mathrm{kHz}$ to $1 \mathrm{~Hz}$ in steps of $100 \mathrm{mV}$ with a 2 min rest at each potential of interest before each measurement to allow for equilibration. Anodic and cathodic scans were performed from the OCP as suggested by Lockett et al. with a 30 min wait period at OCP between scans. ${ }^{17}$ All differential capacitance data were reported at $46 \mathrm{~Hz}$, as that was deemed an appropriate frequency for all EMImTFSI/solvent mixtures studied. Using this protocol, experiments were performed in duplicate and the calculated values of $C_{\min }$ were within $10 \%$ of one another; the $C-V$ curves were in good agreement.

A conductivity meter (CDM83, Radiometer Copenhagen) with a compensation probe was used to measure the conductivity of the RTIL/organic solvent mixtures. Select mixtures were sheared on a rheometer (Anton Parr MCR 501, U.S.A.) in a Couette cell to independently measure the viscosity of the mixtures. All measurements were performed at room temperature, $21 \pm 2{ }^{\circ} \mathrm{C}$.

\section{ASSOCIATED CONTENT}

\section{S Supporting Information}

Viscosity measurements of the EMImTFSI/solvent mixtures. Cyclic voltammograms of as-received and purified EMImTFSI. Frequency-dependent differential capacitance analysis for determining the appropriate frequency at which to compare capacitance values. Capacitance-voltage profiles for EMImTF$\mathrm{SI} /$ solvent mixtures showing hysteresis. Details of the coarsegrained simulations. The Supporting Information is available free of charge on the ACS Publications website at DOI: 10.1021/acs.jpclett.5b00899.

\section{AUTHOR INFORMATION}

\section{Corresponding Author}

*E-mail: iaksay@princeton.edu.

\section{Notes}

The authors declare no competing financial interest.

\section{ACKNOWLEDGMENTS}

This work was supported in part by a Multidisciplinary University Research Initiative (MURI) through the Air Force Office of Scientific Research (Grant \#FA9550-13-1-0004) and in part by an ARPA-E grant through the Department of Energy (Grant \#DE-AR0000319). D.T.L. was supported by the Princeton Center for Theoretical Science. N.J.S. was supported in part by the Lidow Senior Thesis Fund of the School of Engineering and Applied Science at Princeton University. P.G.D. acknowledges the support of the National Science 
Foundation (Grant Nos. CBET-1263565 and CHE-1213343). The authors would like to thank R. K. Prud'homme for the use of his rheometer and B. Wilson for his helpful instruction.

\section{REFERENCES}

(1) Armand, M.; Endres, F.; MacFarlane, D. R.; Ohno, H.; Scrosati, B. Ionic-Liquid Materials for the Electrochemical Challenges of the Future. Nat. Mater. 2009, 8, 621-629.

(2) Simon, P.; Gogotsi, Y. Materials for Electrochemical Capacitors. Nat. Mater. 2008, 7, 845-854.

(3) Yang, X. W.; Cheng, C.; Wang, Y. F.; Qiu, L.; Li, D. LiquidMediated Dense Integration of Graphene Materials for Compact Capacitive Energy Storage. Science 2013, 341, 534-537.

(4) Zhu, Y.; Murali, S.; Stoller, M. D.; Ganesh, K. J.; Cai, W.; Ferreira, P. J.; Pirkle, A.; Wallace, R. M.; Cychosz, K. A.; Thommes, M.; et al. Carbon-Based Supercapacitors Produced by Activation of Graphene. Science 2011, 332, 1537-1541.

(5) Arulepp, M.; Permann, L.; Leis, J.; Perkson, A.; Rumma, K.; Jänes, A.; Lust, E. Influence of the Solvent Properties on the Characteristics of a Double Layer Capacitor. J. Power Sources 2004, 133, 320-328.

(6) McEwen, A. B.; Ngo, H. L.; LeCompte, K.; Goldman, J. L. Electrochemical Properties of Imidazolium Salt Electrolytes for Electrochemical Capacitor Applications. J. Electrochem. Soc. 1999, 146, 1687-1695.

(7) Ruiz, V.; Huynh, T.; Sivakkumar, S. R.; Pandolfo, A. G. Ionic Liquid-Solvent Mixtures as Supercapacitor Electrolytes for Extreme Temperature Operation. R. Soc. Chem. Adv. 2012, 2, 5591-5598.

(8) Pope, M. A.; Korkut, S.; Punckt, C.; Aksay, I. A. Supercapacitor Electrodes Produced through Evaporative Consolidation of Graphene Oxide-Water-Ionic Liquid Gels. J. Electrochem. Soc. 2013, 160, A1653-A1660.

(9) Feng, G.; Huang, J.; Sumpter, B. G.; Meunier, V.; Qiao, R. A "Counter-Charge Layer in Generalized Solvents" Framework for Electrical Double Layers in Neat and Hybrid Ionic Liquid Electrolytes. Phys. Chem. Chem. Phys. 2011, 13, 14723-14734.

(10) Li, S.; Zhang, P.; Fulvio, P. F.; Hillensheim, P. C.; Feng, G.; Dai, S.; Cummigs, P. T. Enhanced Performance of Dicationic Ionic Liquid Electrolytes by Organic Solvents. J. Phys.: Condens. Matter 2014, 26, 284105.

(11) McEwen, A. B.; McDevitt, S. F.; Koch, V. R. Nonaqueous Electrolytes for Electrochemical Capacitors: Imidazolium Cations and Inorganic Fluorides with Organic Carbonates. J. Electrochem. Soc. 1997, 144, L84-L86.

(12) Merlet, C.; Salanne, M.; Rotenberg, B.; Madden, P. A. Influence of Solvation on the Structural and Capacitive Properties of Electrical Double Layer Capacitors. Electrochim. Acta 2013, 101, 262-271.

(13) Fedorov, M. V.; Kornyshev, A. A. Ionic Liquids at Electrified Interfaces. Chem. Rev. 2014, 114, 2978-3036.

(14) Grahame, D. C. The Electrical Double Layer and the Theory of Electrocapillarity. Chem. Rev. 1947, 41, 441-501.

(15) Liu, W.; Yan, X.; Lang, J.; Xue, Q. Effects of Concentration and Temperature of $\mathrm{EMIMBF}_{4} /$ Acetonitrile Electrolyte on the Supercapacitive Behavior of Graphene Nanosheets. J. Mater. Chem. 2012, 22, $8853-8861$.

(16) Siinor, L.; Siimenson, C.; Ivaništšev, V.; Lust, K.; Lust, E. Influence of Cation Chemical Composition and Structure on the Double Layer Capacitance for $\mathrm{Bi}(111) \mid$ Room Temperature Ionic Liquid Interface. J. Electroanal. Chem. 2012, 668, 30-36.

(17) Lockett, V.; Sedev, R.; Ralston, J.; Horne, M.; Rodopoulos, T. Differential Capacitance of the Electrical Double Layer in Imidazolium-Based Ionic Liquids: Influence of Potential, Cation Size, and Temperature. J. Phys. Chem. C 2008, 112, 7486-7495.

(18) Pope, M. A. Electrochemical Double-Layer Capacitors Based on Functionalized Graphene. Ph.D. Thesis, Princeton University, Princeton, NJ, June 2013.
(19) Kornyshev, A. A.; Luque, N. B.; Schmickler, W. Differential Capacitance of Ionic Liquid Interface with Graphite: The Story of Two Double Layers. J. Solid State Electrochem. 2014, 18, 1345-1349.

(20) Zhang, S.; Sun, N.; He, X.; Lu, X.; Zhang, X. Physical Properties of Ionic Liquids: Database and Evaluation. J. Phys. Chem. Ref. Data 2006, 35, 1475-1517.

(21) Nanjundiah, C.; McDevitt, S. F.; Koch, V. R. Differential Capacitance Measurements in Solvent-Free Ionic Liquids at $\mathrm{Hg}$ and $\mathrm{C}$ Interfaces. J. Electrochem. Soc. 1997, 144, 3392-3397.

(22) Chaban, V. V.; Voroshylova, I. V.; Kalugin, O. N.; Prezhdo, O. V. Acetonitrile Boosts Conductivity of Imidazolium Ionic Liquids. J. Phys. Chem. B 2012, 116, 7719-7727.

(23) Fedorov, M. V.; Georgi, N.; Kornyshev, A. A. Double Layer in Ionic Liquids: The Nature of the Camel Shape of Capacitance. Electrochem. Commun. 2010, 12, 749-749.

(24) Aurbach, D. Nonaqueous Electrochemistry; Marcel Dekker Inc.: New York, 1999.

(25) Grousson, M.; Tarjus, G.; Viot, P. Monte Carlo Study of the Three-Dimensional Coulomb Frustrated Ising Ferromagnet. Phys. Rev. E 2001, 64, 9.

(26) Limmer, D. T. Interfacial Ordering and Unbounded Capacitance at Ionic Liquid-Metal Interfaces. arXiv.org, e-print Arch. Condens. Matter 2015, No. arXiv: 1506.02667.

(27) Wen, R.; Rahn, B.; Magnussen, O. M. Potential-Dependent Adlayer Structure and Dynamics at the Ionic Liquid/Au(111) Interface: A Molecular-Scale In Situ Video-STM Study. Angew. Chem., Int. Ed. 2015, 54, 6062-6066.

(28) Uysal, A.; Zhou, H.; Feng, G.; Lee, S. S.; Li, S.; Cummings, P. T.; Fulvio, P. F.; Dai, S.; McDonough, J. K.; Gogotsi, Y.; Fenter, P. Interfacial Ionic 'Liquids': Connecting Static and Dynamic Structures. J. Phys.: Condens. Matter 2015, 27, 9.

(29) Bard, A. J., Faulkner, Larry, R. Electrochemical Methods: Fundamentals and Applications; John Wiley \& Sons, Inc.: New York, 2000. 